\title{
Piecewise Constant Control Set Systems
}

\author{
Andrej V. Plotnikov ${ }^{1,2, *}$, Anastasiya V. Arsirii ${ }^{2}$ \\ ${ }^{1}$ Department Applied Mathematics, Odessa State Academy Civil Engineering and Architecture, Odessa, 65029, Ukraine \\ ${ }^{2}$ Department Optimal Control, Odessa National University, Odessa, 65026, Ukraine
}

\begin{abstract}
In this article we prove that for any measurable admissible control $w(\cdot)$ and for any $\varepsilon>0$ there exists piecewise constant admissible control $\bar{w}(\cdot)$ such that for set solutions of control set system are $\varepsilon$-neighbouring.
\end{abstract}

Keywords Set Differential Equation, Control System, Piecewise Constant Control

\section{Introduction}

In recent years the development of the calculus in metric spaces has attracted some attention[1-7]. Earlier, F.S. de Blasi, F. Iervolino[8] started the investigation of set differential equations (SDEs) in semilinear metric spaces. This has now evolved into the theory of SDEs as an independent discipline: properties of solutions[1-3,5,36], the impulse equations[1,2,37], control systems[38-41] and asymptotic methods[1-3,42-46]. On the other hand, SDEs are useful in other areas of mathematics. For example, SDEs are used, as an auxiliary tool, to prove existence results for differential inclusions[1,26,31,35]. Also, one can employ SDEs in the investigation of fuzzy differential equations[2,6,21-23, $25,26]$. Moreover, SDEs are a natural generalization of the usual ordinary differential equations in finite (or infinite) dimensional Banach spaces.

In many engineering control systems piecewise constant controls, instead of measurable controls are applied. In this article we prove that for any measurable admissible control $w(\cdot)$ and for any $\varepsilon>0$ there exists piecewise constant admissible control $\bar{w}(\cdot)$ such that for set solutions of control set system are $\varepsilon$-neighbouring.

\section{Preliminaries}

Let $\operatorname{comp}\left(R^{n}\right)\left(\operatorname{conv}\left(R^{n}\right)\right)$ be a set of all nonempty (convex) compact subsets from the space $R^{n}$,

$$
h(A, B)=\min _{r \geq 0}\left\{S_{r}(A) \supset B, S_{r}(B) \supset A\right\}
$$

be Hausdorff distance between sets $A$ and $B, S_{r}(A)$ is $r$-neighborhood of set $A$.

Let $A, B, C$ be in $\operatorname{conv}\left(R^{n}\right)$. The set $C$ is the Hukuhara difference of $A$ and $B$, if $B+C=A$, i.e.

$$
C=A \stackrel{H}{-B}
$$

* Corresponding author:

a-plotnikov@ukr.net (Andrej V. Plotnikov)

Published online at http://journal.sapub.org/ajcam

Copyright @ 2011 Scientific \& Academic Publishing. All Rights Reserved
From Radstrom's Cancellation Lemma[47], it follows that if this difference exists, then it is unique.

Definition 1[48]. A mapping $X:[0, T] \rightarrow \operatorname{conv}\left(R^{n}\right)$ is differentiable in the sense of Hukuhara at $t \in[0, T]$ if for some $\delta>0$ the Hukuhara differences

$$
X(t+\Delta) \frac{H}{} X(t), \quad X(t) \frac{H}{} X(t-\Delta)
$$

exists in $\operatorname{conv}\left(R^{n}\right)$ for all $0<\Delta<\delta$ and there exists an $D X(t) \in \operatorname{conv}\left(R^{n}\right)$ such that

$$
\begin{gathered}
\lim _{\Delta \rightarrow 0_{+}} h\left(\Delta^{-1}\left(X(t+\Delta) \frac{H}{X} X(t)\right), D X(t)\right)=0 \text { and } \\
\lim _{\Delta \rightarrow 0_{+}} h\left(\Delta^{-1}\left(X(t) \frac{H}{X} X(t-\Delta)\right), D X(t)\right)=0
\end{gathered}
$$

Here $D X(t)$ is called the Hukuhara derivative of $X(t)$ at $t$.

Consider the Cauchy problem with small parameter

$$
D X=A(t) X+G(t), \quad X(0)=X_{0},
$$

where $A(t)$ is $(n \times n)$-dimensional matrix-valued function; $G: R_{+} \rightarrow \operatorname{conv}\left(R^{n}\right)$ is the set-valued map, $X_{0} \in \operatorname{conv}\left(R^{n}\right)$.

Definition 2. A mapping $X:[0, T] \rightarrow \operatorname{conv}\left(R^{n}\right)$ is a solution to the problem (1) if and only if it is continuous and satisfies the integral equation

$$
X(t)=X_{0}+\int_{0}^{t}[A(s) X(s)+G(s)] d s
$$

for all $t \in[0, T]$. Here the integral is understood in the sense of[48] (the integral exists for example if $X($.$) is$ measurable and the real mapping $t \rightarrow h(X(t),\{0\})$ is integrable on $I \subset R_{+}$).

Theorem 1[2]. Let the following conditions are true:

1) $A(\cdot)$ is measurable on $[0, T]$;

2) There exists $a>0$ such that $\|A(t)\| \leq a$ for almost every $t \in[0, T]$;

3) The set-valued map $G:[0, T] \rightarrow \operatorname{conv}\left(R^{n}\right)$ is measurable on $[0, T]$;

4) There exists $g(\cdot) \in L_{2}[0, T]$ such that 
$h(G(t),\{0\}) \leq g(t)$ almost everywhere on $[0, T]$.

Then problem (1) has on $[0, T]$ exactly one solution.

\section{The Control Set Differential Equation}

We consider following control set differential equation

$$
D X=A(t) X+F(t, w), \quad X(0, w)=X_{0},
$$

where $w \in R^{m}$ is the control, $F: R_{+} \times R^{m} \rightarrow \operatorname{conv}\left(R^{n}\right)$ is the set-valued map.

Let $W: R_{+} \rightarrow \operatorname{conv}\left(R^{m}\right)$ be the measurable set-valued map.

Definition 3. The set $L W$ of all measurable single-valued branches of the set-valued map $W(\cdot)$ is the set of the admissible controls.

Obviously, the control set differential equation (2) turns into the ordinary set differential equation

$$
D X=A(t) X+G(t), \quad X(0)=X_{0},
$$

if the control $\widetilde{w}(\cdot) \in L W$ is fixed and $G(t) \equiv F(t, \widetilde{w}(t))$.

Let $X(t)$ denotes the set solution of the differential equation (3), then $X(t, w)$ denotes the set solution of the control differential equation (2) for the fixed $w(\cdot) \in L W$.

Definition 4. The set $Y(T)=\{X(T, w): w(\cdot) \in L W\}$ be called the attainable set of the system (2).

Theorem 2[49]. Let the following conditions are true:

1) $A(\cdot)$ is measurable on $[0, T]$;

2) There exists $a>0$ such that $\|A(t)\| \leq a$ for almost every $t \in[0, T]$;

3) The set-valued map $W:[0, T] \rightarrow \operatorname{conv}\left(R^{m}\right)$ is measurable on $[0, T]$;

4) The set-valued map $F:[0, T] \times R^{m} \rightarrow \operatorname{conv}\left(R^{n}\right)$ satisfies the conditions
a) measurable in $t$;
b) continuous in $w$;

5) There exist $v(\cdot) \in L_{2}[0, T]$ and $l(\cdot) \in L_{2}[0, T]$ such that

$$
h(W(t),\{0\}) \leq v(t), h(F(t, w),\{0\}) \leq l(t)
$$

almost everywhere on $[0, T]$ and all $w \in W(t)$;

6) The set $Q(t)=\{F(t, w(t)): w(\cdot) \in L W\}$ is compact and convex for almost every $t \in[0, T]$.

Then for every $w(\cdot) \in L W$ there exists the set solution $X(\cdot, w)$ on $[0, T]$ and the attainable set $Y(T)$ is compact and convex.

$$
\text { Let } U=\prod_{i=1}^{m}\left[u_{\min }^{i}, u_{\max }^{i}\right] \text { and } W(t) \equiv U \text { on }[0, T] \text {. }
$$

Now, we need to establish that for any measurable admissible control $w(\cdot)$ and for any $\varepsilon>0$ there exists piecewise constant admissible control $\bar{w}(\cdot)$ such that for set solutions of system (2) holds for all $t \in[0, T]$

$$
h(X(t, w), X(t, \bar{w}))<\varepsilon \text {. }
$$

Theorem 3. Let the conditions of the theorem 2 are true, and

7) There exists constant $\gamma>0$ such that

$$
h\left(\int_{0}^{t} F\left(s, w_{1}(s)\right) d t, \int_{0}^{t} F\left(s, w_{2}(s)\right) d s\right) \leq \gamma\left\|\int_{0}^{t} w_{1}(s) d s-\int_{0}^{t} w_{2}(s) d s\right\|
$$

for all $w_{1}(\cdot), w_{2}(\cdot) \in L W$ and $t \in[0, T]$.

Then for every $w(\cdot) \in L W$ there exists $\bar{w}(\cdot) \in L W$ such that

1) $\bar{w}(t)$ is constant on every $\left[(i-1) \frac{T}{k}, i \frac{T}{k}\right], i=\overline{1, k}$;

2) $\bar{w}_{i}(t)=\left\{\left(\bar{w}_{i}^{1}(t), \ldots, \bar{w}_{i}^{m}(t)\right)^{T} \mid \bar{w}_{i}^{j}(t) \in\left\{u_{\min }^{j}, u_{\max }^{j}\right\}, i=\overline{1, k}\right.$,

$j=\overline{1, m}\}$ for every $t \in[0, T]$;

3) for all $t \in[0, T]$

$$
h(X(t, w), X(t, \bar{w})) \leq \gamma e^{a T} \frac{T}{2 k}\left\|u_{\max }-u_{\min }\right\|,
$$

where $u_{\min }=\left(u_{\min }^{1}, \ldots, u_{\min }^{m}\right)^{T}, u_{\max }=\left(u_{\max }^{1}, \ldots, u_{\max }^{m}\right)^{T}$.

Proof. We have any $w(\cdot) \in L W$ and any $k \in N$. Let $W_{i}=\left(W_{i}^{1}, \ldots, W_{i}^{m}\right)^{T}$, where $W_{i}^{j}=\int_{0}^{i \frac{T}{k}} w^{j}(s) d s, i=\overline{1, k}, j=\overline{1, m}$.

Obviously,

$$
\begin{aligned}
& W_{i+1}^{j}-W_{i}^{j}=\int_{0}^{\frac{(i+1) T}{k}} w^{j}(s) d s-\int_{0}^{\frac{i T}{k}} w^{j}(s) d s=\int_{\frac{i T}{k}}^{\frac{(i+1) T}{k}} w^{j}(s) d s, \\
& u_{\min }^{j} \frac{T}{k} \leq W_{i+1}^{j}-W_{i}^{j} \leq u_{\max }^{j} \frac{T}{k}, j=\overline{1, m},
\end{aligned}
$$

and

$$
\left\|W_{i+1}-W_{i}\right\| \leq\left\|u_{\max }-u_{\min }\right\| \frac{T}{k} .
$$

Now we obtain

$$
\bar{w}(t)=\left\{\begin{array}{cc}
\bar{w}_{1}, & \quad t \in\left[0, \frac{T}{k}\right) \\
\bar{w}_{k-1}, & t \in\left[\frac{(k-2) T}{k}, \frac{(k-1) T}{k}\right), \\
\bar{w}_{k}, & t \in\left[\frac{(k-1) T}{k}, T\right]
\end{array}\right.
$$

such that

1) $\bar{w}_{1}=\left(\bar{w}_{1}^{1}, \ldots, \bar{w}_{1}^{m}\right)$, where

$$
\bar{w}_{1}^{j}=\left\{\begin{array}{l}
u_{\max }^{j}, \quad \text { if } W_{1}^{j} \geq \frac{T}{2 k}\left(u_{\max }^{j}+u_{\min }^{j}\right) \\
u_{\min }^{j}, \quad \text { if } W_{1}^{j}<\frac{T}{2 k}\left(u_{\max }^{j}+u_{\min }^{j}\right)
\end{array}, j=\overline{1, m} ;\right.
$$

2) $\bar{w}_{i}=\left(\bar{w}_{i}^{1}, \ldots, \bar{w}_{i}^{m}\right)$, where

$$
\bar{w}_{i}^{j}=\left\{\begin{array}{ll}
u_{\max }^{j}, & \text { if } \quad W_{i}^{j}-\sum_{l=1}^{i-1} \bar{w}_{l}^{j} \frac{T}{k} \geq \frac{T}{2 k}\left(u_{\max }^{j}+u_{\min }^{j}\right) \\
u_{\min }^{j}, & \text { if } \quad W_{i}^{j}-\sum_{l=1}^{i-1} \bar{w}_{l}^{j} \frac{T}{k}<\frac{T}{2 k}\left(u_{\max }^{j}+u_{\min }^{j}\right)
\end{array},\right.
$$


$j=\overline{1, m}$.

Obviously, for $i=1$ and $j=\overline{1, m}$ we have

a) if $\bar{w}_{1}^{j}=u_{\max }^{j}$, then

$$
\begin{gathered}
W_{1}^{j}-\bar{w}_{1}^{j} \frac{T}{k} \leq 0, W_{2}^{j}-\bar{w}_{1}^{j} \frac{T}{k} \geq \frac{T}{2 k}\left(u_{\max }^{j}-u_{\min }^{j}\right), \\
W_{2}^{j} \leq W_{1}^{j}+u_{\max }^{j} \frac{T}{k}, 0 \geq W_{1}^{j}-\bar{w}_{1}^{j} \frac{T}{k} \geq-\frac{T}{2 k}\left(u_{\max }^{j}-u_{\min }^{j}\right) ;
\end{gathered}
$$

b) if $\bar{w}_{1}^{j}=u_{\min }^{j}$, then

$$
\begin{gathered}
W_{1}^{j}-\bar{w}_{1}^{j} \frac{T}{k} \geq 0, W_{2}^{j}-\bar{w}_{1}^{j} \frac{T}{k} \leq \frac{T}{2 k}\left(u_{\max }^{j}-u_{\min }^{j}\right), \\
W_{2}^{j} \geq W_{1}^{j}+u_{\max }^{j} \frac{T}{k}, 0 \leq W_{1}^{j}-\bar{w}_{1}^{j} \frac{T}{k} \leq \frac{T}{2 k}\left(u_{\max }^{j}-u_{\min }^{j}\right) .
\end{gathered}
$$

Hence we obtain

$$
\left|W_{1}^{j}-\bar{w}_{1}^{j} \frac{T}{k}\right| \leq \frac{T}{2 k}\left(u_{\max }^{i}-u_{\min }^{j}\right),
$$

and

$$
\left\|W_{1}-\bar{w}_{1} \frac{T}{k}\right\| \leq \frac{T}{2 k}\left\|u_{\max }-u_{\min }\right\| .
$$

Thus, by induction, we obtain that, for $i=\overline{2, k}$ and $j=\overline{1, m}$

$$
\begin{gathered}
\left|W_{i}^{j}-\sum_{l=1}^{i} \bar{w}_{l}^{j} \frac{T}{k}\right| \leq \frac{T}{2 k}\left(u_{\max }^{i}-u_{\min }^{j}\right), \quad j=\overline{1, m} \text {, and } \\
\left\|W_{i}-\sum_{l=1}^{i} \bar{w}_{l} \frac{T}{k}\right\| \leq \frac{T}{2 k}\left\|u_{\max }-u_{\min }\right\| .
\end{gathered}
$$

Therefore, if $t_{i}=\frac{i T}{k}, i=\overline{1, k}$; then

$$
\left\|\int_{o}^{t_{s}} w(s) d s-\int_{0}^{t_{i}} \bar{w}(s) d s\right\| \leq \frac{T}{2 k}\left\|u_{\max }-u_{\min }\right\| .
$$

Now, we take $t \in\left(\frac{(i-1) T}{k}, \frac{i T}{k}\right)$. Then

$\left\|\int_{o}^{t} w(s) d s-\int_{0}^{t} \bar{w}(s) d s\right\| \leq\left\|W_{i-1}-\sum_{l=1}^{i-1} \bar{w}_{l} \frac{T}{k}+\int_{\frac{(i-1) T}{k}}^{t}\left(w(s)-\bar{w}_{i}\right) d s\right\|$.

As for all $j=\overline{1, m}$

$$
\begin{aligned}
& W_{i}^{j}-\sum_{l=1}^{i} \bar{w}_{l}^{j} \frac{T}{k} \geq W_{i-1}^{j}-\sum_{l=1}^{i-1} \bar{w}_{l}^{j} \frac{T}{k}+\int_{\frac{(i-1) T}{k}}^{t}\left(w(s)-\bar{w}_{i}^{j}\right) d s \geq \\
& \geq W_{i-1}^{j}-\sum_{l=1}^{i-1} \bar{w}_{l}^{j} \frac{T}{k}
\end{aligned}
$$

then

$$
\left\|\int_{o}^{t} w(s) d s-\int_{0}^{t} \bar{w}(s) d s\right\| \leq \max \left\{\left\|W_{i}-\sum_{l=1}^{i} \bar{w}_{l} \frac{T}{k}\right\|,\left\|W_{i-1}-\sum_{l=1}^{i-1} \bar{w}_{l} \frac{T}{k}\right\|\right\} .
$$

By (4), we get

$$
\left\|\int_{0}^{t} w(s) d s-\int_{0}^{t} \bar{w}(s) d s\right\| \leq \frac{T}{2 k}\left\|u_{\max }-u_{\min }\right\|
$$

for all $t \in[0, T]$.

Now, applying definition 2 and condition 7 of the theorem, we obtain

$$
\begin{gathered}
h(X(t, w), X(t, \bar{w}))=h\left(\int_{0}^{t}[A(s) X(s, w)+F(s, w(s))] d s,\right. \\
\left.\int_{0}^{t}[A(s) X(s, \bar{w})+F(s, \bar{w}(s))] d s\right) \leq \int_{0}^{t} h(A(s) X(s, w), A(s) X(s, \bar{w}))+ \\
+h\left(\int_{0}^{t} F(s, w(s)) d s, \int_{0}^{t} F(s, \bar{w}(s)) d s\right) \leq \\
\leq a \int_{0}^{t} h(X(s, w), X(s, \bar{w})) d s+\gamma \mid \int_{0}^{t} w(s) d s-\int_{0}^{t} \bar{w}(s) d s \|
\end{gathered}
$$

Using Gronwall-Bellman's inequality, we obtain

$$
h(X(t, w), X(t, \bar{w})) \leq \gamma e^{a T}\left\|\int_{0}^{t} w(s) d s-\int_{0}^{t} \bar{w}(s) d s\right\| .
$$

By (5), we have

$$
h(X(t, w), X(t, \bar{w})) \leq \gamma e^{a T} \frac{T}{2 k}\left\|u_{\max }-u_{\min }\right\| .
$$

Theorem is proved.

Remark. Obviously, if we take $k>\gamma e^{a T} \frac{T}{2 \varepsilon}\left\|u_{\max }-u_{\min }\right\|$; then $h(X(t, w), X(t, \bar{w}))<\varepsilon$ for all $t \in[0, T]$.

\section{Conclusions}

Here we used the approach of Hukuhara at definition of the derivative which has essential shortages. However the given approach is well investigated by many authors. Also in the literature exist other approaches to definition of the derivative[2,3,9,21,29,36], but also they have the shortages. It is easily possible to show that this outcome will be true for some other cases with little changes.

Also we remark that this result helps to build $\varepsilon_{\text {-optimal }}$ piecewise constant controls for optimal control set system (Mayer problem, time-optimal problem and other).

\section{REFERENCES}

[1] N. A. Perestyuk, V. A. Plotnikov, A. M. Samoilenko and N. V. Skripnik, Differential equations with impulse effects: multivalued right-hand sides with discontinuities. De Gruyter Studies in Mathematics. Berlin/Boston: Walter De Gruyter GmbH\&Co., 2011, vol. 40

[2] A. V. Plotnikov and N. V. Skripnik, Differential equations with "clear" and fuzzy multivalued right-hand sides. Asymptotics Methods. AstroPrint, Odessa, 2009

[3] V. A. Plotnikov, A. V. Plotnikov and A. N. Vityuk, Differential equations with a multivalued right-hand side: Asymptotic methods. AstroPrint, Odessa, 1999

[4] L. Ambrosio and L. Tilli, Topics on analysis in metric spaces. Oxford Lecture Series in Mathematics and its Applications. Oxford University Press, Oxford, 2004, vol. 25 
[5] V. Lakshmikantham, T. Granna Bhaskar and J. Vasundhara Devi, Theory of set differential equations in metric spaces. Cambridge Scientific Publishers. 2006

[6] V. Lakshmikantham and R. N. Mohapatra, Theory of Fuzzy Differential Equations and Inclusions. London, Taylor \& Francis, 2003

[7] Plotnikov, A.V. and Skripnik, N.V., 2011, Set-valued differential equations with generalized derivative., J. Advanced Research in Pure Mathematics, 13(1), 144-160

[8] de Blasi, F. S. and Iervolino, F., 1969, Equazioni differentiali con soluzioni a valore compatto convesso., Boll. Unione Mat. Ital., 2(4-5), 491-501

[9] Bede, B. and Stefanini, L., 2008, Generalized Hukuhara differentiability of interval-valued functions and interval differential equations., Univ. Urbino "Carlo Bo", Working Paper Series in Economics, Math. and Statistics. WP-EMS\#2008/03

[10] de Blasi, F. S. and Iervolino, F., 1971, Euler method for differential equations with set-valued solutions., Boll. Unione Mat. Ital., 4(4), 941-949

[11] Brandao Lopes Pinto, A. J., de Blasi, F. S. and Iervolino, F., 1970, Uniqueness and existence theorems for differential equations with compact convex valued solutions., Boll. Unione Mat. Ital., (4), 534-538

[12] Dabrowska, R.. and Janiak, T., 1993, Stability of functionaldifferential equations with compact convex valued solutions., Discuss. Math., (13), 87-92

[13] Drici, Z., Mcrae, F. A. and Vasundhara Devi, J., 2005, Set differential equations with causal operators., Mathematical Problems in Engineering, 2005:2, 185-194

[14] Galanis, G. N., Gnana Bhaskar, T., Lakshmikantham, V., and Palamides, P. K., 2005, Set value functions in Frechet spaces: Continuity, Hukuhara differentiability and applications to set differential equations., J. Nonlinear Analysis, 61, $559-575$

[15] Galanis, G. N., Tenali, G. B. and Lakshmikantham, V., 2008, Set differential equations in Frechet spaces., J. Appl. Anal., $14,103-113$

[16] Gnana Bhaskar, T. and Lakshmikantham, V., 2003, Set differential equations and flow invariance., Appl. Anal. (82), $357-368$

[17] Gnana Bhaskar, T. and Lakshmikantham, V., 2004, Lyapunov stability for set differential equations., Dynam. Systems Appl., (13), 1-10

[18] Gnana Blaskar, T. and Vasundhara Devi, J., 2005, Set differential systems and vector Lyapunov functions., Appl. Math. Comput., 165(3), 539-548

[19] Kisielewicz, M., 1975, Description of a class of differential equations with set-valued solutions., Lincei-Rend. Sc. fis. mat. e nat., $58,158-162$

[20] Kisielewicz, M., Serafin, B. and Sosulski, W., 1975, Existence theorem for functional-differential equation with compact convex valued solutions., Demonstratio math., 13(2), 229-237

[21] Komleva, T. A., Plotnikov, A. V. and Skripnik, N. V., 2008,
Differential equations with set-valued solutions., Ukr. Math. J., 60(10), 1540-1556

[22] Lakshmikantham, V., 2004, The connection between set and fuzzy differential equations., Facta Univ. Ser. Mech. Automat. Control. Robot., 4, 1-10

[23] Lakshmikantham, V., 2005, Set differential equations versus fuzzy differential equations., J. Applied Mathematics and Computation, 164, 277-294

[24] Laksmikantham, V., Leela, S. and Vatsala, A. S., 2002, Setvalued hybrid differential equations and stability in terms of two measures., J. Hybrid Systems, (2), 169-187

[25] Laksmikantham, V., Leela, S. and Vatsala, A. S., 2003, Interconnection between set and fuzzy differential equations., Nonlinear Anal., 54, 351-360

[26] Lakshmikantham, V. and Tolstonogov, A. A., 2003, Existence and interrelation between set and fuzzy differential equations., Nonlinear Anal., 55, 255-268

[27] Piszczek, M., 2006, Second Hukuhara derivative and cosine family of linear set-valued functions., An. Acad. Paedagogicae Cracoviensis. Studia Math., 33, 87-98

[28] Piszczek, M., 2008, On a multivalued second order differential problem with Hukuhara derivative., Opuscula Math., 28(2), 151-161

[29] Plotnikov, A. V., 2000, Differentiation of multivalued mappings. T-derivative., Ukr. Math. J., 52(8), 1282-1291

[30] Plotnikov, A. V. and Tumbrukaki, A. V., 2000, Integro-differential equations with multivalued solutions., Ukr. Math. J., 52(3), 413-423

[31] Plotnikova, N. V., 2006, Approximation of a set of solutions of linear differential inclusions., Nonlinear Oscil., 9(3), 375-390

[32] Skripnik, N. V., 2008, The Krasnoselskii-Krein theorem for differential equations with multivalued solutions., Visn. Khark. Univ., Ser. Mat. Prykl. Mat. Mekh., 826(58), 87-99

[33] Smajdor, A., 2003, On a multivalued differential problem., Internat. J. Bifur. Chaos Appl. Sci. Engrg., 13, 1877-1882

[34] Tise, I., 2007, Data dependence of the solutions for set differential equations., Carpathian J. Math., 23(1-2), 192-195

[35] A. Tolstonogov, Differential inclusions in a Banach space, Dordrecht, Kluwer Academic Publishers, 2000

[36] Vityuk, A. N., 2003, Differential equations of fractional order with set-valued solutions., Visn. Odes. Derzh. Univ., Ser. Fiz.-Mat. Nauky., 8(2), 108-112

[37] Ahmad, B. and Sivasundaram, S., 2008, $\varphi_{0}$-stability of impulsive hybrid setvalued differential equations with delay by perturbing lyapunov functions., Communications in Applied Analysis, 12(2), 137-146

[38] Arsirii, A. V. and Plotnikov, A. V., 2009, Systems of control over set-valued trajectories with terminal quality criterion., Ukr. Math. J., 61(8), 1349-1356

[39] N. D. Phu and T. T. Tung, Multivalued Differential Equations, VNU - HCM City: Publishing House, 2005

[40] Phu, N. D. and Tung, T. T., 2007, Existence of solutions of 
set control differential equations., J. Sci. Tech. Devel., 10(6), $5-14$

[41] A. V. Plotnikov, "Differential inclusions with Hukuhara derivative and some control problems," Rep. VINITI 26.04.82, 2036-82, 1982

[42] Janiak, T. and Łuczak-Kumorek, E., 2003, Method on partial averaging for functional-differential equations with Hukuhara's derivative. Studia Univ. "Babeş-Bolyai", Math., XLVIII(2), 65-72

[43] Kichmarenko, O. D., 2009, Averaging of differential equations with Hukuhara derivative with maxima., Int. J. Pure Appl. Math., 57(3), 447-457

[44] Kisielewicz, M., 1976, Method of Averaging for Differential Equations with Compact Convex Valued Solutions., Rend. Math., 9(3), 397-408
[45] Plotnikov, V. A. and Kichmarenko, O. D., 2006, Averaging of controlled equations with the Hukuhara derivative., Nonlinear Oscil., 9(3), 365-374

[46] Plotnikov, V. A. and Rashkov, P. I., Averaging in differential equations with Hukuhara derivative and delay., Funct. Differ. Equ., (8), 371-381

[47] Radstrom, H, 1952, An embeldding theorem for spaces of convex sets., Proc. Amer. Math. Soc., (3), 165-169

[48] Hukuhara, M., 1967, Integration des applications mesurables dont la valeur est un compact convexe., Funkcial. Ekvac., (10), 205-223

[49] Plotnikov, A.V., Komleva, T.A. and Arsiry, A.V., 2009, Necessary and sufficient optimality conditions for a control fuzzy linear problem, Int. J. Industrial Mathematics, 1(3), 197-207 